\title{
Pattern of Coronary Artery Stenosis among Ischaemic Heart Disease Cases in Chittagong
}

\author{
Iqbal $\mathrm{AT}^{1}$, Uddin $\mathrm{J}^{2}$, Banik $\mathrm{D}^{3}$, Salehuddin ${ }^{4}$, Mamun $\mathrm{H}^{5}$, Murad $\mathrm{H}^{6}$
}

\begin{abstract}
Many studies were conducted on the topic over the whole world but there is none in Chittagong, Bangladesh. To know the pattern of coronary artery stenosis in Chittagong we have conducted the study because it is important for effective case management. It was an observational study. Convenient sampling technique was used and sample size was fixed to 110 considering resource constraints. All the cases were diagnosed on the basis of history, clinical features and laboratory investigations. Coronary artery angiogram was methodically conducted. All relevant data had been recorded and were managed manually. The findings were validated statistically. Discussion was made with updated literature review and finally conclusion was drawn. Total 110 cases were studied. Stenosis was found in $77(70 \%$ ) cases. Among them $83 \%$ were male and $17 \%$ were female. Age range was $30-80$ years but $76 \%$ cases were of 40-60 years age group. Among the stenosed cases SVD 29\%, DVD 20\% and TVD 20\% only. Only $01 \%$ was LMCA. Commonest stenosed vessel was LAD $71 \%$. RCA 60\%, LCX 58\% and LMCA 6\%. 47\% of stenosed cases were found with normal ECG. Ejection fraction of $57 \%$ stenosed cases was $>55 \%$. Study results are not significantly apart from studies in home and abroad. The limitation is small sample size.
\end{abstract}

1. Corresponding Author: Dr. Abu Tarek Iqbal Associate Professor, Department of Cardiology Chattagram Maa-O-Shishu Hospital Medical College e-mail: dr.tarekiqbal@yahoo.com

2. Dr. Jalal Uddin Professor, Department of Community Medicine Chattagram Maa-O-Shishu Hospital Medical College

3. Dr. Dhiman Banik

Associate Professor, Department of Cardiology National Heart Foundation

4. Dr. Salehuddin

Assistant Professor, Department of Cardiology Barishal Medical College

5. Dr. Hasan Mamun Associate Professor, Department of Cardiology Chattagram Maa-O-Shishu Hospital Medical College

6. Dr. Hasan Murad Assistant Professor, Department of Cardiology USTC
So, a multicenter study on a large scale cases is hereby advocated for a conclusive opinion.

Keywords: Pattern, Coronary artery stenosis, Chittagong.

\section{Introduction}

Coronary artery stenosis is the narrowing of coronary artery due to deposition of fat in coronary artery. It obstructs blood flow to myocardium which is manifested by angina, heart failure and sudden death. The problem is attributed by modifiable and non-modifiable risk factors. Modifiable risk factors are: Diabetes mellitus, Hypertension, Smoking, Sedentary life style etc. and nonmodifiable risk factors are: Age, Sex, personality type-A, Ethnicity etc. ${ }^{1}$ Coronary artery stenosis is a global problem. It claims $30 \%$ lives in developed countries. In developing countries the problem has been increasing day by day $^{2}$. Clinical features of the disease depends on severity of stenosis. More stenosis more severity of clinical features and in some cases patient may die without prior notice i.e in first episode. However periodic check up and screening program may predict it earlier and accordingly corrective measures can prevent premature death $^{3}$. For diagnosis of coronary artery stenosis clinical features and supporting laboratory investigations like ECG, ETT and Echocardiography are enough. Angiogram is needed for detection of exact location and extent of stenosis $^{1}$. Main objective of the study is to see the pattern of coronary artery stenosis among the classically diagnosed ischaemic heart disease cases in Chittagong region.

\section{Materials and Methods}

It was an observational study. The study was conducted in a private cardiac center of Chittagong during July to December 2014. Classically diagnosed IHD cases were selected for angiography (IHD diagnosis was on the basis of history, clinical features and lab investigations: ECG, Echo, ETT etc.). Patients with ejection fraction $<35 \%$, creatinine $>2 \mathrm{mg} \%$ and of over 80 years were excluded. Angiogram was methodically done (Coronary angiography was performed by the Judkin's technique through femoral artery access). The angiographic characters include lesion location and percentage of stenosis. Interpretations of angiogram were visually estimated by 2 cardiologists. Whole procedure and findings were recorded in a pretested format. Sampling 
technique and sample size was conveniently fixed considering resource constraints. Recorded data was managed manually. Results were contrasted with previous studies. Z-Test was used in this context.

\section{Results}

Age range was $30-80$ years. $76 \%$ cases were of $40-60$ years age group, $18 \%$ over 60 and $06 \%$ below 40 years age group. Male $83 \%$ and female $17 \%$. $60 \%$ of stenosed cases were found with normal BMI. Top 3 modifiable risk factors were: Hypertension 73\%, Diabetes $56 \%$ and Smoking 45\%. Angiography was done in 110 cases. Among them 30\% were no vessel disease (No stenosis). SVD 29\%, DVD 18\%, TVD 22\% and LMCA $01 \%$. Among the stenosed vessels LAD ranked first (71\%). RCA $60 \%$, LCX 53\% and LMCA $6.5 \%$ respectively. ECG findings was normal among $47 \%$ of stenosed cases and Ejection fraction of $57 \%$ stenosed cases was $>55 \%$.

\section{Table-I: Age, Sex structure of respondents}

\begin{tabular}{ll}
\hline \multicolumn{1}{c}{ Variables } & No. of cases (\%) \\
\hline 1. Age of respondents & \\
$<40$ years & $05(06 \%)$ \\
$40-60$ years & $58(76 \%)$ \\
$>60$ years & $14(18 \%)$ \\
Total & $77(100 \%)$ \\
2. Sex of respondents & \\
Male & $64(83 \%)$ \\
Female & $13(17 \%)$ \\
Total & $77(100 \%)$ \\
\hline
\end{tabular}

Source: Study report

Table-II: Number of stenosed vessels

\begin{tabular}{lc}
\hline No. of stenosed vessels & Frequency (\%) \\
\hline Normal coronary artery ( No stenosis) & $33(30 \%)$ \\
Single vessel stenosis (SVD) & $32(29 \%)$ \\
Double vessel stenosis (DVD) & $20(18 \%)$ \\
Triple vessels stenosis (TVD) & $24(22 \%)$
\end{tabular}

Left main coronary artery(LMCA) stenosis $01(01 \%)$

Total

$110(100 \%)$

Source: Study report
Table-III: Ranking of stenosed vessels

\begin{tabular}{ll}
\hline Name of stenosed vessels & Frequency (\%) \\
\hline Left anterior descending artery (LAD) & $55(71 \%)$ \\
Right coronary artery (RCA) & $46(60 \%)$ \\
Left circumflex artery (LCX) & $41(53 \%)$ \\
Left main coronary artery (LMCA) & $05(6.5 \%)$ \\
\hline
\end{tabular}

Source: Study report

\section{Discussion}

Age of occurrence of coronary artery stenosis, sex variation are similar $(\mathrm{P}>.05)$ with existing literature4 i.e 40-60 years are more risky 58 (76\%) and male preponderance is there $64(83 \%)$. Ranking of modifiable risk factors like hypertension, diabetes and smoking are a bit different with other studies. According to Nazmul and Hoque the ranking was Smoking, Hypertension, Diabetes $^{4}$. The difference in ranking is due to site and sample variation. Present study shows Normal coronary artery $33(30 \%)$. This is significantly high $(\mathrm{P}<.05)$ in comparison to other studies in abroad. According to Ibrahim et al no vessel stenosis is $6.9 \%$. This difference indicates our poor clinical assessment of patients. Rate of SVD, DVD and TVD in present study are $29 \%, 18 \%$ and $22 \%$ respectively. Study conducted by Ibrahim et al are $18 \%, 26 \%$, and $45 \%$. According to present study LAD $71 \%$, RCA $60 \%$ and LCX 53\% which were $42 \%, 32 \%$ and $26 \%$ according to Ibrahim shah et al. Significant difference $(\mathrm{P}<.05)$ between two studies may be due to variation of sample size ( 110 vs 1325 ), ethnicity and observer variation etc ${ }^{5}$

In conclusion, angiography is the gold standard test for detection of exact location of stenosis and number of vessels affected. It also shows severity of stenosis but it is not hassle free. So, case selection should be judicious considering all relevant aspects.

\section{References}

1. WHO (2004). Cardio technical series-771:1050.

2. Stamler J. Prevalence of CHD: An overview. New England Journal of Medicine.2005;502:870-72.

3. Park J E. Text book of preventive and social medicine. 19th edition. Jabalpur(India): Banarashidas bhanot;2008.

4. Alam N, Haque KMA. Prevalence of coronary heart disease among rural people with risk factor analysis. The Orion. 2007;26:436-37.

5. Ibrahim S. Clinical profile, angiographic characteristics and treatment recommendations in patient with CAD. JPMS.2013;3(2):94-100 . 